\title{
DSMC-LBM mapping scheme for rarefied and non-rarefied gas flows
}

\author{
G. Di Staso ${ }^{a, *}$, H.J.H. Clercx ${ }^{\mathrm{a}}$, S. Succi ${ }^{\mathrm{b}, \mathrm{c}}$, F. Toschi ${ }^{\mathrm{a}, \mathrm{b}, \mathrm{d}}$ \\ ${ }^{a}$ Department of Applied Physics, Eindhoven University of Technology, \\ Den Dolech 2, 5600 MB, Eindhoven, Netherlands \\ ${ }^{b}$ Istituto per le Applicazioni del Calcolo, Consiglio Nazionale delle Ricerche, \\ Via dei Taurini 19, 00185, Rome, Italy \\ ${ }^{c} J o h n$ A. Paulson School of Engineering and Applied Sciences, Harvard University, \\ 29 Oxford Street, Cambridge MA 02138, USA \\ ${ }^{d}$ Department of Mathematics and Computer Science, Eindhoven University of Technology, \\ Den Dolech 2, 5600 MB, Eindhoven, Netherlands
}

\begin{abstract}
We present the formulation of a kinetic mapping scheme between the Direct Simulation Monte Carlo (DSMC) and the Lattice Boltzmann Method (LBM) which is at the basis of the hybrid model used to couple the two methods in view of efficiently and accurately simulate isothermal flows characterized by variable rarefaction effects. Owing to the kinetic nature of the LBM, the procedure we propose ensures to accurately couple DSMC and LBM at a larger Kn number than usually done in traditional hybrid DSMC—Navier-Stokes equation models. We show the main steps of the mapping algorithm and illustrate details of the implementation. Good agreement is found between the moments of the single particle distribution function as obtained from the mapping scheme and from independent LBM or DSMC simulations at the grid nodes where the coupling is imposed. We also show results on the application of the hybrid scheme based on a simpler mapping scheme for plane Poiseuille flow at finite Kn number. Potential gains in the computational efficiency assured by the application of the coupling scheme are estimated for the same flow.
\end{abstract}

\footnotetext{
* Corresponding author

Email addresses: g.di.staso@tue.nl, +31 402472143 (G. Di Staso), h.j.h.clercx@tue.nl (H.J.H. Clercx), succi@iac.cnr.it (S. Succi), f.toschi@tue.nl (F. Toschi)
}

Preprint submitted to Journal of Computational Science

April 22, 2016

(C) 2016. This manuscript version is made available under the Elsevier user license http://www.elsevier.com/open-access/userlicense/1.0/ 
Keywords: kinetic theory, Grad's moments method, non-equilibrium effects, rarefied gas flows, hybrid method

2010 MSC: 76P05, 82B40, 82B80

\section{Introduction}

Research in gas flows characterized by a large range of scales and by disparate levels of non-equilibrium effects poses a challenge to statistical physics modelling and rises interest in industry for simulating flows in micro-, nanoelectromechanical systems and in material processing tools $[1-3$. The extent of the departure of a flow from the equilibrium state is traditionally measured in terms of the Knudsen number:

$$
\mathrm{Kn}=\frac{\lambda}{\ell} \approx \frac{\lambda}{Q}\left|\frac{d Q}{d \ell}\right|,
$$

where $\lambda$ is the gas mean free path, $\ell$ is the smallest hydrodynamic characteristic scale and $Q$ is a fluid dynamic quantity of interest such as the gas pressure, velocity, temperature [4. According to the Knudsen number, the gas flows can be classified into the hydrodynamic $(\mathrm{Kn}<0.01)$, slip $(0.01<\mathrm{Kn}<0.1)$, transition $(0.1<\mathrm{Kn}<10)$ and free molecular regime $(\mathrm{Kn}>10)$. The kinetic description of gases based on the Boltzmann equation, valid at any $\mathrm{Kn}$, allows to cover flow conditions from the very rarefied to the hydrodynamic limit [5]. The two limits, rarefied and continuum, have traditionally been studied numerically by approximating the Boltzmann equation via the Direct Simulation Monte Carlo (DSMC) [6] or by solving the Navier-Stokes equations which can be derived from truncation at first order of the Chapman-Enskog procedure 7 . While the DSMC method is particularly suited to rarefied gas flow (transitional regime), its computational costs make it unpractical to study hydrodynamic flows 2. Conversely, the continuum description of the flow provided by solving the Navier-Stokes equations and applying the no-slip boundary condition is not accurate whenever $\mathrm{Kn}>0.01$ [8]. Corrections to the boundary conditions of Navier-Stokes equations such as to reproduce the velocity slip and temperature jump at the gas-surface interface in case of slip flow regime are often 
not accurate and may also predict incorrect qualitative behavior of the flow [9, 10. Moreover, the derivation of extended hydrodynamic equations employing higher-order Chapman-Enskog approximations (Burnett and super-Burnett equations) have shown limited success [8]. Alternatively, macroscopic transport equations can be originated from moments expansion methods such as the

25 Grad's method [11, 12. However, difficulties in imposing boundary conditions for those moments without a clear physical meaning, as well complexity in the resulting systems of equations prevent the application of the method for the simulation of flows of industrial interest.

It is therefore evident that whenever the flow presents a large range of $\mathrm{Kn}$, due to the current computational and modelling limitations of the available methods, a multiscale hybrid model has to be used.

When dealing with multiscale models, domain decomposition techniques represent the most natural way to handle the problem. Within this approach, the domain is decomposed according to a continuum breakdown parameter between 35 regions where continuum-level macroscopic equations (either Euler or NavierStokes equations) are valid and regions where substantial non-equilibrium effects are present and kinetic methods, typically DSMC, are needed (see Refs. 8, 1319]). Then a special treatment is imposed to couple the flow fields in the areas of overlap between the different regions, e.g. [20 22].

40 For completeness, the domain decomposition technique is not the only method adopted in the literature as alternative approaches are proposed. For example in 23, the Boltzmann equation is solved for a short period of time to obtain the rate of change of the average flow variables which are then used to update the continuum-level velocity field. In [24, instead, macroscopic equations are modified so to include effects due to kinetic contributions which take into account perturbations from the equilibrium state of the velocity distribution.

The approach that we introduce here follows the domain decomposition technique as commonly done in models proposed in literature but it departs from those as the flow at the continuum level and at moderate rarefied conditions is simulated with the Lattice Boltzmann Method (LBM). 
Moreover, since it has been largely demonstrated that LBM, due to its intrinsic kinetic nature, is an accurate and efficient numerical solver not only for flows at Navier-Stokes description level but also for flows at finite Kn number (see Refs. [25] 37]), the present model has the advantage, over the other hybrid methods which use traditional Navier-Stokes solvers, that the need for using the computationally expensive DSMC solver can be postponed to larger values of Kn. This is equivalent to say that the size of the domain where DSMC solver is still needed can be significantly reduced, thus improving the overall computational efficiency of the simulation.

In this work we principally focus on the most delicate aspect of any hybrid coupling model, i.e. the two-way extraction and transfer of information at the interface between the two numerical methods. The mapping schemes we developed, in fact, allow to pass from DSMC to LBM domains and vice versa correctly transferring also the non-equilibrium information. The amount of non-equilibrium information that can be passed is then essentially determined by the LB model and in particular by the chosen set of discrete velocities and the isotropy conditions the set is able to fulfill.

Simulations performed to validate the mapping scheme show that an accurate transfer of information is achievable for flows up to $\mathrm{Kn}=0.25$ for a 39 -points 70 Gauss-Hermite quadrature with sixth-order isotropy (D3Q39), under the assumption that the flow is isothermal, i.e. no external heat source is present, and viscous dissipation or other thermal effects are negligible.

Finally, to check functionality of the DSMC-LBM hybrid model and assess its computational efficiency, tests, based on a simpler mapping scheme, are also performed showing, for the particular simulated flow, a significant speed-up with respect to a full DSMC simulation.

\section{Mapping schemes}

Since both LB and DSMC are widely documented in the literature, only a few basic aspects are discussed in this paper. For an exhaustive treatment 
about DSMC and LBM methods, the reader should refer to [6] and [38.

Both methods aim to determine the fluid motion as described by the Boltzmann equation. The main feature which clearly distinguishes the LBM from the DSMC, is the reduction of the degrees of freedom of the velocity space. In fact in LBM particles at each lattice site $\mathbf{x}$ can only propagate along a finite number of directions with an assigned speed $\boldsymbol{\xi}_{a}$, while in DSMC the velocity space is not constrained to a set of discrete velocities.

Before introducing the mapping scheme between the DSMC and LBM, we note that in order to quantitatively reproduce DSMC solutions for finite-Kn number flows, the LB model needs three basic ingredients:

90 1. kinetic boundary conditions, [37, 39,43 ;

2. higher-order lattice (HOL), 29, 44;

3. regularization procedure, [29, 45].

Few words on the two lattices used in this work are in order. The conventional D3Q19 LB model, satisfying isotropy conditions up to the fourth order, is able to describe the flow up to the Navier-Stokes level of description. In order for LB to accurately simulate rarefied gas flows, it is essential that the set of discrete velocities satisfies higher order isotropy conditions. In this work we shall take a step further, beyond the standard D3Q19 model, requiring that the lattice satisfies isotropy conditions up to the sixth order. A lattice able to fulfil this requirement, and still maintaining the Cartesian coordinate implementation is the D3Q39 model as described in 44. In Table 1 the D3Q39 lattice discrete velocities $\boldsymbol{\xi}_{a}$ and weights $w_{a}$ are collected. For comparison, the same is done also for the D3Q19 model.

The main idea at the basis of the mapping scheme is that the single particle distribution function $f(\mathbf{x}, \boldsymbol{\xi}, t)$ can be expanded in terms of the dimensionless Hermite orthonormal polynomials, $\mathcal{H}(\boldsymbol{\xi})$, in the velocity space $\boldsymbol{\xi}$ as [11, 12, 44]:

$$
f(\mathbf{x}, \boldsymbol{\xi}, t)=\omega(\boldsymbol{\xi}) \sum_{n=0}^{\infty} \frac{1}{n !} \mathbf{a}^{(n)}(\mathbf{x}, t) \mathcal{H}^{(n)}(\boldsymbol{\xi}),
$$




\begin{tabular}{|c|c|c|c|}
\hline LB model & $\boldsymbol{\xi}_{a}$ & $w_{a}$ & $a$ \\
\hline \multirow{7}{*}{ D3Q19 } & $(0,0,0)$ & $1 / 3$ & 0 \\
\hline & $( \pm 1,0,0)$ & $1 / 18$ & $1-2$ \\
\hline & $(0, \pm 1,0)$ & $1 / 18$ & $3-4$ \\
\hline & $(0,0, \pm 1)$ & $1 / 18$ & $5-6$ \\
\hline & $( \pm 1, \pm 1,0)$ & $1 / 36$ & $7-10$ \\
\hline & $( \pm 1,0, \pm 1)$ & $1 / 36$ & $11-14$ \\
\hline & $(0, \pm 1, \pm 1)$ & $1 / 36$ & $15-18$ \\
\hline \multirow[t]{14}{*}{ D3Q39 } & $(0,0,0)$ & $1 / 12$ & 0 \\
\hline & $( \pm 1,0,0)$ & $1 / 12$ & $1-2$ \\
\hline & $(0, \pm 1,0)$ & $1 / 12$ & $3-4$ \\
\hline & $(0,0, \pm 1)$ & $1 / 12$ & $5-6$ \\
\hline & $( \pm 1, \pm 1, \pm 1)$ & $1 / 27$ & $7-14$ \\
\hline & $( \pm 2,0,0)$ & $2 / 135$ & $15-16$ \\
\hline & $(0, \pm 2,0)$ & $2 / 135$ & $17-18$ \\
\hline & $(0,0, \pm 2)$ & $2 / 135$ & $19-20$ \\
\hline & $( \pm 2, \pm 2,0)$ & $1 / 432$ & $21-24$ \\
\hline & $( \pm 2,0, \pm 2)$ & $1 / 432$ & $25-28$ \\
\hline & $(0, \pm 2, \pm 2)$ & $1 / 432$ & $29-32$ \\
\hline & $( \pm 3,0,0)$ & $1 / 1620$ & $33-34$ \\
\hline & $(0, \pm 3,0)$ & $1 / 1620$ & $35-36$ \\
\hline & $(0,0, \pm 3)$ & $1 / 1620$ & $37-38$ \\
\hline
\end{tabular}

Table 1: Sets of discrete velocities and weights for the D3Q19 model (lattice speed of sound, $\left.c_{s}^{2}=1 / 3\right)$ and the D3Q39 model $\left(c_{s}^{2}=2 / 3\right)$. 
where $\omega(\boldsymbol{\xi})$ is the weight function associated with the Hermite polynomials, and $\mathbf{a}^{(n)}$ are the rank- $n$ tensors representing the dimensionless expansion coefficients defined as:

$$
\mathbf{a}^{(n)}=\int f(\mathbf{x}, \boldsymbol{\xi}, t) \mathcal{H}^{(n)}(\boldsymbol{\xi}) d \boldsymbol{\xi} .
$$

The first coefficients of the series, due to the definition of the Hermite polynomials (see Appendix B for the definition of Hermite polynomials), can be identified as the hydrodynamic moments (or a combination of those) of the distribution $f(\mathbf{x}, \boldsymbol{\xi}, t)$ :

$$
\begin{gathered}
\mathbf{a}^{(0)}=\int f(\mathbf{x}, \boldsymbol{\xi}, t) \mathcal{H}^{(0)}(\boldsymbol{\xi}) d \boldsymbol{\xi}=\int f(\mathbf{x}, \boldsymbol{\xi}, t) d \boldsymbol{\xi}=\rho, \\
\mathbf{a}^{(1)}=\int f(\mathbf{x}, \boldsymbol{\xi}, t) \mathcal{H}^{(1)}(\boldsymbol{\xi}) d \boldsymbol{\xi}=\int f(\mathbf{x}, \boldsymbol{\xi}, t) \boldsymbol{\xi} d \boldsymbol{\xi}=\rho \mathbf{u} \\
\mathbf{a}^{(2)}=\int f(\mathbf{x}, \boldsymbol{\xi}, t) \mathcal{H}^{(2)}(\boldsymbol{\xi}) d \boldsymbol{\xi}=\int f(\mathbf{x}, \boldsymbol{\xi}, t)\left(\boldsymbol{\xi} \boldsymbol{\xi}-c_{s}^{2} \boldsymbol{\delta}\right) d \boldsymbol{\xi}=\mathbf{P}+\rho\left(\mathbf{u u}-c_{s}^{2} \boldsymbol{\delta}\right) \\
\mathbf{a}^{(3)}=\int f(\mathbf{x}, \boldsymbol{\xi}, t) \mathcal{H}^{(3)}(\boldsymbol{\xi}) d \boldsymbol{\xi}=\int f(\mathbf{x}, \boldsymbol{\xi}, t)\left(\boldsymbol{\xi} \xi \boldsymbol{\xi}-c_{s}^{2} \boldsymbol{\xi} \delta\right) d \boldsymbol{\xi}= \\
\mathbf{Q}+\mathbf{u a}^{(2)}-(D-1) \rho \mathbf{u u u}
\end{gathered}
$$

and analogously for higher-order coefficients. In Eqs. (6) and (7), $\delta$ represents the Kronecker delta function while $\mathbf{P}$ and $\mathbf{Q}$ represent the full second and third order moments which have the usual appearance if the intrinsic velocity, $\mathbf{c}=$ $\boldsymbol{\xi}-\mathbf{u}(\mathbf{x}, t)$, is used:

$$
\begin{gathered}
\mathbf{P}=m \int f \operatorname{cc} d \mathbf{c} \\
\mathbf{Q}=m \int f \operatorname{ccc} d \mathbf{c}
\end{gathered}
$$

Due to the orthonormality of the Hermite polynomials,

$$
f(\mathbf{x}, \boldsymbol{\xi}, t) \approx f^{N}(\mathbf{x}, \boldsymbol{\xi}, t)=\omega(\boldsymbol{\xi}) \sum_{n=0}^{N} \frac{1}{n !} \mathbf{a}^{(n)}(\mathbf{x}, t) \mathcal{H}^{(n)}(\boldsymbol{\xi})
$$


and $f^{N}(\mathbf{x}, \boldsymbol{\xi}, t)$ has the same leading $N$ velocity moments as the complete

It has to be noted that the following procedures can be extended to any suitable LB stencil whose discrete speeds are actually abscissae of a Gauss-Hermite quadrature.

\subsection{DSMC2LB mapping scheme}

Firstly, we present the DSMC2LB projection step. As a remark, the main characteristic of this coupling step is that, instead of directly using information from DSMC particles, namely their velocities, we chose to adopt a different approach which uses the DSMC hydrodynamic moments, and Grad's formalism, to determine the discrete non-equilibrium distribution functions to be advanced in time by the LB solver. In this way, not only the coupling step is fully consistent with the LB Grad's formalism, but it has also the advantage to reduce possible stability issues related to the large fluctuations inherent to the DSMC representation. More considerations on stability issues are given in Section 2.3 In correspondence with the DSMC cells/LBM nodes where the coupling occurs, the cumulative averages of the DSMC hydrodynamic variables, properly scaled (see Appendix A on how to perform such scaling), are used to compute the coefficients $\mathbf{a}_{\mathrm{DSMC}}^{(n)}$ of the truncated distribution $f_{\mathrm{DSMC}}^{N}(\mathbf{x}, \boldsymbol{\xi}, t)$ in Eq. 10 .

We now take advantage of the fact that the distribution $f_{\mathrm{DSMC}}^{N}(\mathbf{x}, \boldsymbol{\xi}, t)$ can be completely and uniquely determined by its values at a set of discrete velocities 
and, if the Gauss-Hermite quadrature is used, then the coefficients $\mathbf{a}_{\mathrm{DSMC}}^{(n)}$ can be expressed as:

$$
\begin{gathered}
\mathbf{a}_{\mathrm{DSMC}}^{(n)}=\int f_{\mathrm{DSMC}}^{N}(\mathbf{x}, \boldsymbol{\xi}, t) \mathcal{H}^{(n)}(\boldsymbol{\xi}) d \boldsymbol{\xi}= \\
\sum_{a=0}^{d-1} \frac{w_{a}}{\omega\left(\boldsymbol{\xi}_{a}\right)} f_{\mathrm{DSMC}}^{N}\left(\mathbf{x}, \boldsymbol{\xi}_{a}, t\right) \mathcal{H}^{(n)}\left(\boldsymbol{\xi}_{a}\right),
\end{gathered}
$$

where $w_{a}$ and $\boldsymbol{\xi}_{a}$ are the weights and abscissae of a Gauss-Hermite quadrature of algebraic precision of degree $\geq 2 N$, and $d$ is the total number of discrete velocities of the quadrature.

The definitions of the first hydrodynamic moments in the LBM are:

$\rho=\sum_{a} f_{a}, \rho \mathbf{u}=\sum_{a} f_{a} \boldsymbol{\xi}_{a}, \quad \mathbf{P}=\sum_{a} f_{a} \boldsymbol{\xi}_{a} \boldsymbol{\xi}_{a}-\rho \mathbf{u u}, \quad \mathbf{Q}=\sum_{a} f_{a} \boldsymbol{\xi}_{a} \boldsymbol{\xi}_{a} \boldsymbol{\xi}_{a}-\rho \mathbf{u u u}$

and similarly for higher-order moments.

Comparing Eq. 111 with Eq. (12) and recalling the definitions of the Hermite polynomials $\mathcal{H}^{(n)}$ and that the coefficients $\mathbf{a}^{(n)}$ are the velocity moments of the $f^{N}(\mathbf{x}, \boldsymbol{\xi}, t)$, or a proper combination of those, it is immediate to see that the discrete distributions are the scaled values of the continuous distribution function evaluated at the Gauss-Hermite quadrature abscissae $\boldsymbol{\xi}_{a}$ :

$$
f_{\mathrm{DSMC} 2 \mathrm{LB}, a}(\mathbf{x}, t)=\frac{w_{a} f_{\mathrm{DSMC}}^{N}\left(\mathbf{x}, \boldsymbol{\xi}_{a}, t\right)}{\omega\left(\boldsymbol{\xi}_{a}\right)} .
$$

In essence, once the $f_{\mathrm{DSMC}}^{N}(\mathbf{x}, \boldsymbol{\xi}, t)$ is built from the DSMC hydrodynamic moments according to Eq. 10 and evaluated at the quadrature abscissae,

$f_{\mathrm{DSMC}}^{N}\left(\mathbf{x}, \boldsymbol{\xi}_{a}, t\right)$, the discrete (non-equilibrium) distributions to be supplemented to the LBM solver at the coupling nodes can be computed from Eq. 13.

\subsection{LB2DSMC mapping scheme}

The inverse reconstruction step (LB2DSMC) requires that at the LBM lattice nodes/DSMC cells where the coupling occurs, the velocities of the DSMC particles are sampled from a continuous distribution function.

At those lattice sites, the LBM discrete non-equilibrium functions $f_{\mathrm{LB}, a}$, are 
used to compute the coefficients of the expansion in Eq. (10):

$$
\mathbf{a}_{\mathrm{LB}}^{(n)}=\sum_{a=0}^{d-1} f_{\mathrm{LB}, a}(\mathbf{x}, t) \mathcal{H}^{(n)}\left(\boldsymbol{\xi}_{a}\right)
$$

These allow to build the continuous truncated distribution $f_{\mathrm{LB}}^{N}(\mathbf{x}, \boldsymbol{\xi}, t)$. To generate the velocities of the DSMC particles, the distribution should be sampled. Several algorithms can be employed to this aim. We chose to adopt an acceptance/rejection algorithm similar to the one presented in [22]. However, while in 22] a Chapman-Enskog distribution was sampled, in the present case a Grad's distribution has to be sampled but, nonetheless, most of the steps presented there can be used here.

The Grad's velocity distribution, truncated up to order $N$, can be written as

$$
g^{N}(\mathbf{x}, \boldsymbol{\xi}, t)=g^{(0)}(\boldsymbol{\xi}) G(\mathbf{x}, \boldsymbol{\xi}, t)
$$

where $g^{(0)}(\boldsymbol{\xi})$ is the weight function associated with the Hermite polynomials

$$
g^{(0)}(\boldsymbol{\xi})=\omega(\boldsymbol{\xi})=\frac{1}{(2 \pi)^{D / 2}} \exp \left(-\frac{\xi^{2}}{2}\right)
$$

with $D$ being the dimensionality of the flow problem. Eq. (16) represents also a global Maxwell-Boltzmann distribution at thermodynamic equilibrium (here we set a constant temperature $T=1$ as we are interested in isothermal flows). At thermodynamic equilibrium $G(\mathbf{x}, \boldsymbol{\xi}, t)=1$, while away from that condition, it can be expressed as:

$$
\begin{array}{r}
G(\mathbf{x}, \boldsymbol{\xi}, t)=1+\frac{1}{2 !} \mathbf{a}_{\mathrm{LB}}^{(2)} \mathcal{H}^{(2)}(\boldsymbol{\xi}) \\
+\frac{1}{3 !} \mathbf{a}_{\mathrm{LB}}^{(3)} \mathcal{H}^{(3)}(\boldsymbol{\xi})+\cdots+\frac{1}{N !} \mathbf{a}_{\mathrm{LB}}^{(N)} \mathcal{H}^{(N)}(\boldsymbol{\xi})
\end{array}
$$

The steps followed in the generation of the velocities of DSMC particles are outlined in Table 2. Some comments on those steps. The acceptance/rejection method needs to define an envelope function $\gamma(\boldsymbol{\xi})$ such that $\gamma(\boldsymbol{\xi}) \geq g(\boldsymbol{\xi})$ for any $\xi$. In step 3, an amplitude parameter $C$ is set. In this way it is guaranteed that the function $\gamma(\boldsymbol{\xi})=C g^{(0)}(\boldsymbol{\xi})$ envelops most of the Grad's distribution function below it. The larger this parameter, the less probable is the chance that $G(\mathbf{x}, \boldsymbol{\xi}, t)$ is larger than the envelop function, but at the same time, the smaller 


\section{Sampling acceptance/rejection algorithm} for the Grad's distribution LB2DSMC

1. Compute the coefficients

$$
a_{\mathrm{LB}, i j}^{(2)}=\sum_{a} f_{\mathrm{LB}, a}\left(\xi_{a, i} \xi_{a, j}-\delta_{i j}\right)
$$

and similarly for the higher-order ones

2. Find

$$
M \equiv \max \left(\left|a_{\mathrm{LB}, i j}^{(2)}\right|,\left|a_{\mathrm{LB}, i j k}^{(3)}\right|, \ldots,\left|a_{\mathrm{LB}, i j k \ldots}^{(N)}\right|\right)
$$

3. Set the parameter

$$
C=1+30 M
$$

4. Sample a try particle velocity $\boldsymbol{\xi}_{\text {try }}$ from the Maxwell-Boltzmann distribution $g^{(0)}(\boldsymbol{\xi})$ using e.g. the Box-Müller transformation method

5. Accept the $\boldsymbol{\xi}_{\text {try }}$ if $C \mathcal{R} \leq G\left(\mathbf{x}, \boldsymbol{\xi}_{\text {try }}, t\right)$ with $\mathcal{R}$ a uniform deviate in the interval $[0,1)$ otherwise reject it and go back to step 4

6. Generate the DSMC particle velocity as

$$
\mathbf{v}_{j, \mathrm{LB} 2 \mathrm{DSMC}}=\left(\frac{2 k_{B} T_{\mathrm{DSMC}}}{m_{\mathrm{DSMC}}}\right)^{1 / 2} \boldsymbol{\xi}_{\mathrm{try}}+\mathbf{u}_{\mathrm{LB}}
$$

Table 2: Steps of the sampling acceptance/rejection algorithm for the LB2DSMC reconstruction mapping scheme used to generate the velocities of DSMC particles from LBM data.

the efficiency of the sampling method since the efficiency is equal to $1 / C$. In step 6 , the particle velocity is generated as the sum of the thermal velocity and of the local fluid velocity. In Eq. (21), the thermal velocity is determined according to the temperature value and to the molecular mass of the gas as set in 
the DSMC simulation.

Apart from the velocity, also the number of the DSMC particles, $N_{\text {LB2DSMC }}$, must be set in order to guarantee conservation of mass at the coupling sites so that the density from LBM and the density from DSMC, appropriately scaled, match with each other.

In Figure 1, the schematic showing the main steps involved in both the mapping schemes is drawn.

It is interesting to try to identify sources of inaccuracy in the proposed mapping scheme. In the reconstruction and projection steps, in fact, some information is inevitably lost. In particular, in the LB2DSMC reconstruction step, the truncated distribution, $f_{\mathrm{LB}}^{N}(\mathbf{x}, \boldsymbol{\xi}, t)$, is derived from the discrete distributions, $145 f_{\mathrm{LB}, a}(\mathbf{x}, t)$. This truncated distribution is such that only the first $N$ moments are the same as those of the non-truncated continuous distribution $f(\mathbf{x}, \boldsymbol{\xi}, t)$, with the value of $N$ essentially depending on the particular quadrature used. The moments of order higher than $N$, in fact, will not be the same as those of the original continuous distribution. This, in turn, reflects in the fact that the DSMC particles will be given a velocity which is sampled from a distribution which accurately recovers up to the first $N$ moments. If, then, the sampling process were perfectly able to sample the velocity distribution $f_{\mathrm{LB}}^{N}(\mathbf{x}, \boldsymbol{\xi}, t)$, then also the moments computed from the velocities of the particles would be perfectly reproduced in the limit of an infinite number of independent samples. However, since only a finite number of samples can be obtained, measurements of moments will be affected by statistical noise which will be also present in the discrete distribution functions $f_{\mathrm{DSMC} 2 \mathrm{LB}, a}(\mathbf{x}, t)$.

Analogously, in the DSMC2LB projection step, the loss of information derives from the fact that only the first $N$ moments are used to evaluate the truncated discrete distributions $f_{\mathrm{DSMC} 2 \mathrm{LB}, a}(\mathbf{x}, t)$, while, in principle, the DSMC solution possesses information on all the moments up to $N \rightarrow \infty$. The truncation, again, is performed according to the algebraic degree of accuracy of the particular LB quadrature. To be more precise, this does not imply that moments of order larger than $N$ cannot be evaluated but it means that they are not accurately 

distribution from which to sample the velocities of the DSMC particles, then the source of inaccuracy would be mainly related to the acceptance/rejection algorithm and in particular on the choice of parameter $C$ in Eq. 20 which determines the extension of the envelope function $\gamma(\boldsymbol{\xi})$.

170 has direct access to the temperature field, in order for the LB to accurately simulate also thermal effects, different approaches can be followed. Among those, three main strategies can be envisaged: the double distribution function approach where, besides the usual distribution function for the velocity field, a second distribution function is used for the temperature or internal energy field, [46], and standard lattices are used; the multispeed approach where lattices possessing higher isotropy conditions are used, 47]; the mixed method where the velocity field is studied with the usual LB model while the energy equation is solved by different numerical method, usually finite-difference or finite-volume methods, 48. Within the present multiscale scenario, the most natural among the three aforementioned schemes is the multispeed one, as it derives from the Hermite expansion approach. The extension to non-isothermal flows of the present coupling schemes, however, will be object of future works.

\subsection{Stability issues for the coupling schemes}

When dealing with the DSMC2LB coupling step, concerns about stability issues arise. Those are essentially related to the fact that fluctuations on the DSMC hydrodynamic variables may harm positivity of the LB equilibrium distribution functions. For the particular flows discussed in this paper, as we are interested in the stationary state, we choose the cumulative DSMC moments to build the single particle distribution function of Eq. 10p. This choice, combined with the fact that the studied flow is at low Mach number, practically, staves off the aforementioned risks. For flows where the transient flow is of interest, or for which a stationary state does not exist, the instantaneous DSMC moments should be used in Eq. 10. Also in this more demanding case, positivity of the 
LB2DSMC

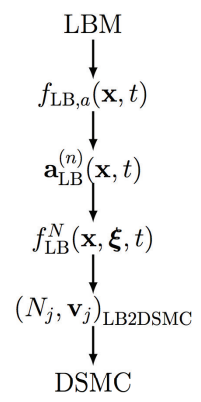

DSMC2LB

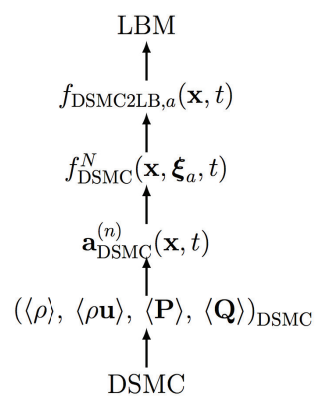

Figure 1: Schematic showing the main steps of the top-down LB2DSMC reconstruction (left) and bottom-up DSMC2LB projection (right) mapping schemes as described in Section 2 and $\mathbf{Q}$ represent the second and third order momentum flux tensors, respectively. Symbol \langle\rangle represents the cumulative average measurements of hydrodynamic moments from the DSMC solver.

LB equilibrium distribution functions can be guaranteed if a sufficiently large number of particles is employed. Fluctuations on hydrodynamic variables can be estimated by applying equilibrium statistical mechanics consideration, see 49. For example, referring to fluctuations of the fluid velocity components within a cell, it is possible to see that the distribution of fluid velocity components realizations follows a normal distribution with standard deviation given by:

$$
\sigma=\sqrt{\frac{k_{B}\langle T\rangle}{m\langle N\rangle}}
$$

185 slightly larger number of DSMC particles than what would be strictly necessary for the method to provide accurate results may be needed. 


\section{Numerical results}

195

\subsection{Comparison between DSMC and LBM data}

To understand and determine the extent of the overlap region where both DSMC and LBM provide comparable accuracy in simulating rarefied gas flows, we performed independent force-driven plane Poiseuille flow simulations with two parallel plates at $x=0$ and $x=H$ and compared results obtained from D3Q19 and D3Q39 LB models with DSMC data. Even if the flow is strictly a monodimensional flow, we used 3D solvers since our final aim is to be able to simulate more complex flows. This choice reflects in the fact that double periodic boundary conditions are imposed along the $y-$ and $z$-directions.

Tests are performed at different Kn number, based on channel height, while keeping constant the Ma number, based on the flow centerline velocity, $u_{\max }$ : $\mathrm{Ma}=u_{\max } / c_{s}=0.1$. The Ma number is set to such a value to guarantee that the lattice equilibria in LBM, expressed as a second-order (D3Q19) or a thirdorder (D3Q39) expansion in Ma number of the local Maxwellian, are positive defined, but it is still sufficiently high to prevent DSMC simulations from becoming impractically computationally expensive.

Moreover, as a result, this allows to treat the flow as a isothermal one. From DSMC simulations, in fact, the temperature increase is never found to exceed $0.5 \mathrm{~K}$, which is fairly negligible as compared to the reference temperature $273 \mathrm{~K}$. In the BGK-LBM simulations, we set the flow Kn number imposing the relaxation time $\tau$ according to the relation [50, 51]:

$$
\tau=\sqrt{\frac{\pi}{8}} \frac{c}{c_{s}} \mathrm{KnH}+0.5
$$

where $c / c_{s}$ is equal to $\sqrt{3}$ for the D3Q19 model and to $\sqrt{3 / 2}$ for the D3Q39 model, and $\mathrm{H}$ is the number of lattice sites along the channel height. Once $\mathrm{Kn}$ and $\mathrm{H}$ are set, $\tau$ is also set. For both D3Q19 and D3Q39 models, kinetic boundary conditions and regularization procedure are applied.

In the DSMC simulations, we set the Kn number imposing the height of the channel, $\mathrm{H}$, and the mean free path $\lambda$. To set $\lambda$, a proper number density $n$ and 


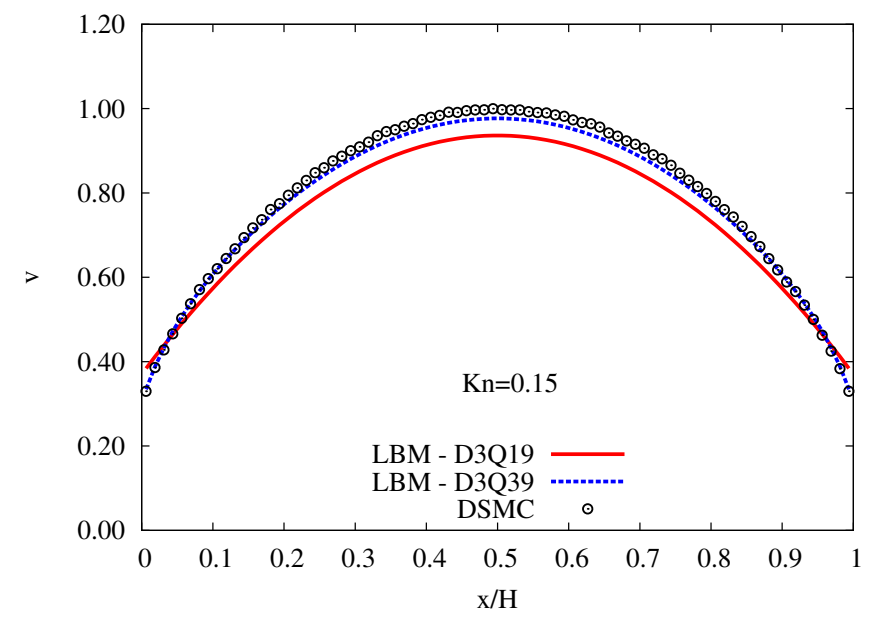

Figure 2: The velocity profiles of the planar force-driven Poiseuille flow for $\mathrm{Kn}=0.15$. The LBM results with both the D3Q19 and D3Q39 models are compared with the DSMC solution. For both the LB models the regularization procedure is applied. Fully diffuse reflection is imposed at the walls, $x / H=0$ and $x / H=1$, for both the LBM and DSMC simulations.

a collision model should be defined. In the case of Hard Sphere (HS) model, the relation between $\lambda$ and $n$ (at equilibrium) is given by [6]:

$$
\lambda=\frac{1}{\sqrt{2} \pi d_{\text {ref }}^{2} n}
$$

where $d_{\text {ref }}$ is a reference molecular diameter. The determination of $\lambda$ from Eq. (24) and estimates on the molecular speed allows to define the space and time discretizations.

Once the number of cells along the channel height is determined from DSMC parameters, an equal number of lattice sites is imposed in the LBM simulation so that the cells centers in DSMC and the LBM lattice sites overlap. In Figure 2 , the velocity profiles along the direction of the forcing, obtained from the LB models and DSMC, normalized with the centerline velocity, are shown for $\mathrm{Kn}=0.15$. The velocity profiles are normalized with the applied forcing $(\rho g)$ and then the DSMC velocity profile is used as a reference for the LB velocity profiles. This is done to acknowledge the different speed of sound in the two 

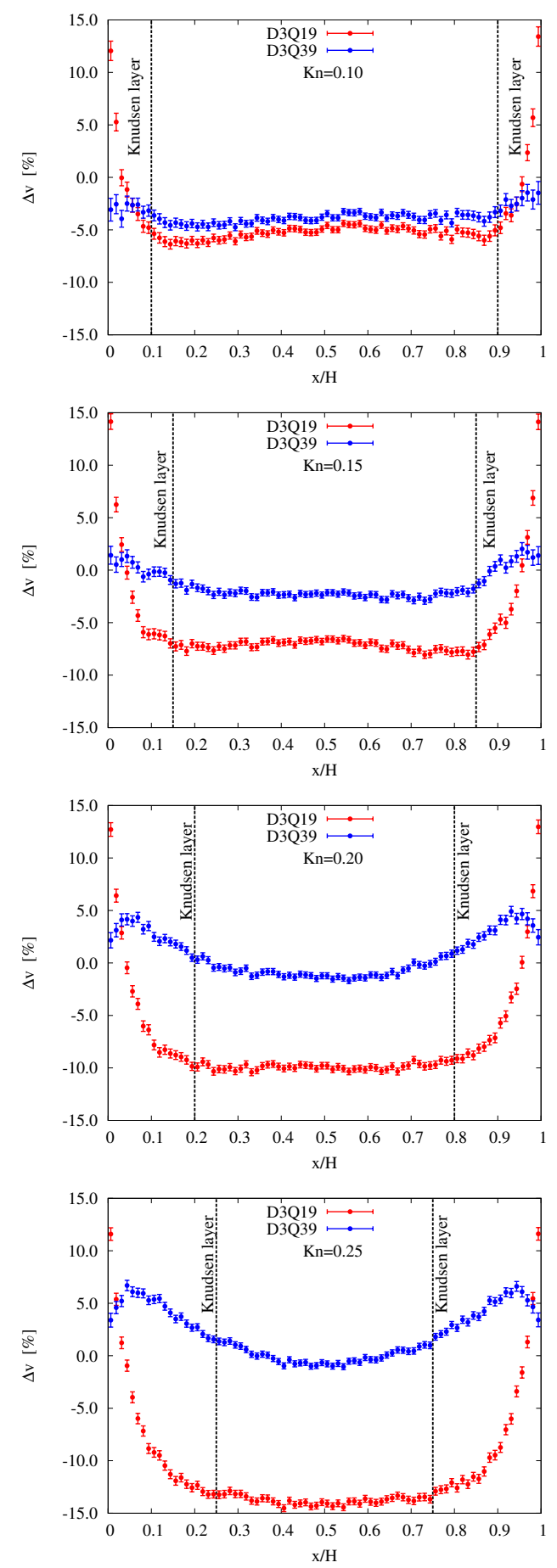

Figure 3: Relative error between LBM results and DSMC data, according to Eq. 26 for $\mathrm{Kn}=0.10-0.25$. Dashed vertical lines represent the boundaries of the Knudsen layer. Error bars from DSMC simulations on fluid velocity are shown. 
lattices.

In the DSMC an Argon-like gas has been simulated and the grid resolution, kept the same for all performed tests, is based on the requirements of a DSMC simulation at $\mathrm{Kn}=0.05$. In all the DSMC simulations, 100 computational molecules are initially placed in each cell of the domain.

LB solution has been considered as converged to the final solution once the following criterion is fulfilled:

$$
\sum_{i} \frac{\left|\mathbf{u}\left(\mathbf{x}_{i}, t\right)-\mathbf{u}\left(\mathbf{x}_{i}, t-1\right)\right|}{\left|\mathbf{u}\left(\mathbf{x}_{i}, t\right)\right|}<10^{-6}
$$

In Eq. (25), $\mathbf{u}\left(\mathbf{x}_{i}, t\right)$ represents the fluid velocity at the lattice nodes at time t. For DSMC, instead, a 1\% fractional error on fluid velocity components is set as the requirement to assume the solution as converged; see Section 5 for the implications in the number of required time steps to achieve such error.

Plots similar to the one of Figure2, have been drawn also for other Kn numbers but they are not reported here. It is more informative, in fact, to inspect the relative errors between DSMC and LBM data as done in Figure 3 . The relative error is defined as:

$$
\Delta v=\frac{v_{L B M}-v_{D S M C}}{v_{L B M}}
$$

and it is shown for simulations at $\mathrm{Kn}=0.10-0.25$.

In the plots of Figure 3, moreover, the boundaries of the Knudsen layer (black dashed vertical lines) are also drawn. The Knudsen layer is a region in proximity of a solid wall which extends within the flow domain up to a distance of the order of one mean free path. Inside this region non-equilibrium effects of the flow are stronger [52, 53. The D3Q19 model, recovering only up to the Navier-Stokes equations level of description, shows to depart from the DSMC solution also within the bulk of the flow and the error increases as the Kn number increases. The D3Q39 model, instead, is able to reproduce the DSMC data to a much better degree of accurary. However, already at $\mathrm{Kn}=0.25$, it is possible to notice some deviations especially within the Knudsen layer as the maximum relative error is about equal to $7.5 \%$. This behavior can be explained taking into account 
that non-equilibrium effects at an order higher than the third may start to play a role.

With this statement, we do not imply that LBM is able to reliably simulate rarefied gas flows only for $\mathrm{Kn} \leq 0.25$, but that with the current LB model we found reasonable agreement with DSMC data up to that Kn number. With larger Gauss-Hermite quadratures, in fact, being able to go beyond the thirdorder in Hermite polynomials expansion guaranteed by the D3Q39, further nonequilibrium effects should be correctly captured. However, we decided not to go further because the next quadrature possessing a high enough algebraic precision to allow an accurate fourth-order in Hermite polynomials expansion involves 91 discrete speeds [54].

\subsection{Numerical results for the DSMC2LB mapping scheme}

Having concluded that the LBM D3Q39 model provides, for the problem at hand, a reasonable accurate solution for $K n \leq 0.25$, we analyze results related to the mapping scheme step that allows to project the DSMC hydrodynamic variables onto the LBM discrete distribution functions for the D3Q39 lattice (DSMC2LB projection step).

${ }_{225}$ To be noted that the unit conversion as delineated in Appendix A to pass from SI units, proper of the DSMC method, to the lattice units, proper of the LB method, is applied during simulations. To validate the procedure outlined in Section 2.1. we ran two sets of independent DSMC and LBM simulations under the same force-driven plane Poiseuille flow with Ma based on the centerline velocity equal to 0.1 and for several Kn numbers. We verified the accordance between the discrete distributions functions as computed from the LBM, $f_{\mathrm{LB}, a}$, and as obtained from the DSMC2LB projection scheme, $f_{\mathrm{DSMC} 2 \mathrm{LB}, a}$, applying Eq. 13p. In Figure 4, a sketch showing the procedure to compare the $f_{\mathrm{LB}, a}$ with the $f_{\mathrm{DSMC} 2 \mathrm{LB}, a}$ is depicted. Data refers to the first fluid node/cell in proximity to the wall located at $x=H$ as shown in the sketch of Figure 5. In Figure6 the ratio $f_{\mathrm{DSMC} 2 \mathrm{LB}, a} / f_{\mathrm{LB}, a}$ is plotted for all discrete speeds $a=0, \ldots, d-1$ and for $\mathrm{Kn}=0.15$ and $\mathrm{Kn}=0.25$. The larger errors that can be detected are about equal 
to $2 \%\left(f_{\mathrm{DSMC} 2 \mathrm{LB}, 36} / f_{\mathrm{LB}, 36} \approx 1.02\right)$ and to $5 \%\left(f_{\mathrm{DSMC} 2 \mathrm{LB}, 36} / f_{\mathrm{LB}, 36} \approx 1.05\right)$ for the simulations at $\mathrm{Kn}=0.15$ and 0.25 , respectively. Most of the other ratios are such that the error is below $1 \%$.

The error bars present in the plots derive from the fact that we use the DSMC hydrodynamic moments to build the truncated distributions $f_{\mathrm{DSMC}}^{N}\left(\mathbf{x}, \boldsymbol{\xi}_{a}, t\right)$ and those are inherently characterized by statistical noise.

We also note that the larger error bars are present for the discrete speeds with larger module. This may be attributed to the fact that the magnitude of the discrete distribution function, $f_{a}$, is smaller the larger the module of the corresponding discrete speed, $\boldsymbol{\xi}_{a}$, while the statistical noise does not depend on the particular discrete speed.

From the comparison of the discrete distributions, $f_{a}$, only, however, it is difficult to understand if the projection mapping scheme is providing accurate results. So it is more informative to compute the hydrodynamic moments from $f_{\mathrm{LB}, a}$ and from $f_{\mathrm{DSMC} 2 \mathrm{LB}, a}$ at the same node depicted in Figure 5 . The first few moments are reported in Table 3 .

It can be seen that a good matching is found always within the error bars. Concluding, the projection mapping scheme is able to pass from the DSMC hydrodynamic quantities to the LBM discrete distributions preserving a reasonable level of accuracy.

\begin{tabular}{cccc}
\hline \hline Kn=0.15 & $\rho$ [l.u.] & $\rho u_{y}[$ l.u.] & $P_{x y}+\rho u_{x} u_{y}[$ l.u.] \\
\hline LBM & 1.0 & 0.0282 & -0.0151 \\
\hline DSMC2LB & $1.002 \pm 0.007$ & $0.0277 \pm 0.0042$ & $-0.0146 \pm 0.0027$ \\
\hline Kn=0.25 & $\rho$ [l.u.] & $\rho u_{y}[$ l.u. $]$ & $P_{x y}+\rho u_{x} u_{y}[$ l.u. $]$ \\
\hline LBM & 1.0 & 0.0352 & -0.0180 \\
\hline DSMC2LB & $0.999 \pm 0.007$ & $0.0340 \pm 0.0042$ & $-0.0167 \pm 0.0024$ \\
\hline \hline
\end{tabular}

Table 3: Comparison between the first few moments as computed from $f_{\mathrm{DSMC} 2 \mathrm{LB}, a}$ obtained from the projection mapping scheme and from the native LBM simulations, $f_{\mathrm{LB}, a}$, at the node depicted in Figure 5 Moments are expressed in lattice units. 


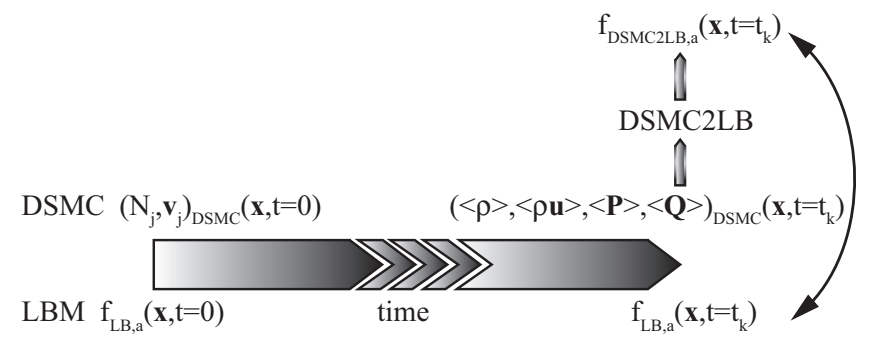

Figure 4: Schematic representing the procedure used to compare the discrete populations built from the DSMC hydrodynamic moments following the projection DSMC2LB algorithm, $f_{\mathrm{DSMC} 2 \mathrm{LB}, a}\left(\mathbf{x}, t=t_{k}\right)$, with native discrete populations obtained from an independent LBM simulation, $f_{\mathrm{LB}, a}\left(\mathbf{x}, t=t_{k}\right)$, under the same flow conditions, namely $\mathrm{Kn}$ and $\mathrm{Ma}$, at time $t=t_{k}$, when the steady-state condition is reached.

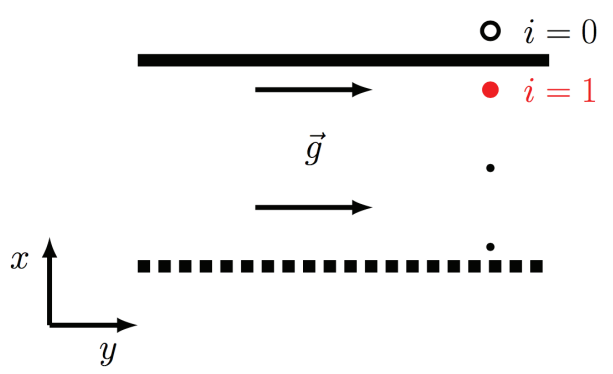

Figure 5: Sketch showing the location of the node (red node at $i=1$ ) where data plotted in Figures 6 and 8 are taken. $\vec{g}$ represents the body force driving the fluid. 

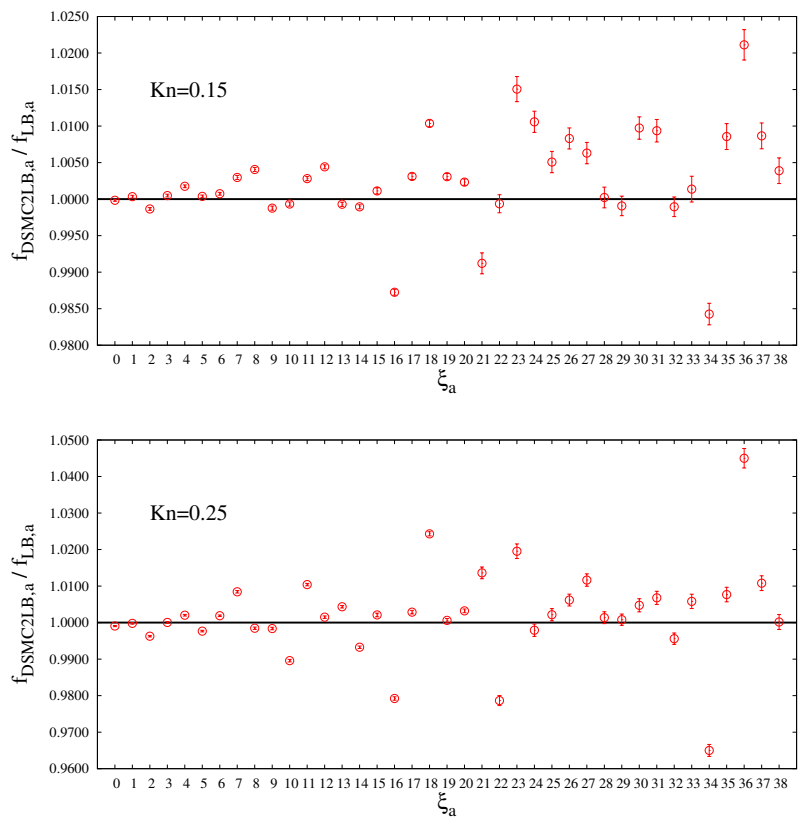

Figure 6: Ratio $f_{\mathrm{DSMC} 2 \mathrm{LB}, a} / f_{\mathrm{LB}, a}$ where $f_{\mathrm{DSMC} 2 \mathrm{LB}, a}$ are computed from Eq. 13 for plane Poiseuille flow at $\mathrm{Kn}=0.15$ (top) and at $\mathrm{Kn}=0.25$ (bottom).

\subsection{Numerical results for the LB2DSMC mapping scheme}

We now move on to analyse the results related to the reconstruction mapping scheme step that allows to reconstruct from the LBM discrete distributions, $f_{\mathrm{LB}, a}$, the continuous truncated distribution function from which the velocities of the DSMC particles can be sampled (LB2DSMC reconstruction step).

The unit conversion as delineated in Appendix A to pass from lattice units, proper of the LB method, to the SI units, proper of the DSMC method, is applied during simulations. As done for the previous step, to validate the procedure outlined in Section 2.2, we ran two independent set of DSMC and LBM simulations under the same force-driven plane Poiseuille flow with $\mathrm{Ma}=0.1$ and for several Kn numbers.

As shown in Figure 7, we compared the velocity distribution functions as obtained from the DSMC simulation collecting the velocities, $\mathbf{v}_{j, \text { DSMC }}$, of the 


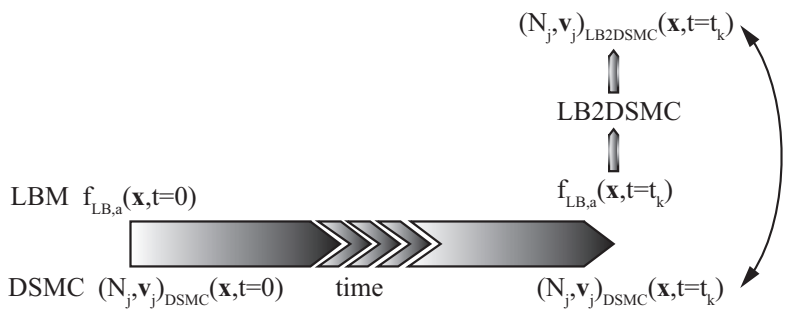

Figure 7: Schematic representing the procedure used to compare the distribution function of the velocity component, $v_{j, \mathrm{DSMC}}$, obtained from a native DSMC simulation with the distribution function of the velocity of the particles, $v_{j, \mathrm{LB} 2 \mathrm{DSMC}}$, obtained from the reconstruction mapping algorithm LB2DSMC, under the same flow conditions, namely Kn and Ma, at time $t=t_{k}$, when the steady-state condition is reached.

particles residing at the cell identified in Figure 5 and as obtained from the velocities of the particles sampled from the velocity distribution function built as in Eq. 15 using the algorithm outlined in Table $2, \mathbf{v}_{j, \text { LB2DSMC. }}$

In Figure 8 in particular, the distributions for the velocity component along the direction of the forcing, $v_{y}$, are compared for $\mathrm{Kn}=0.15$ and $\mathrm{Kn}=0.25$, respectively.

The mean and the standard deviation for the two cases are collected in Table 4 The velocities of the particles are collected for both cases after a steady-state

\begin{tabular}{ccc}
\hline \hline $\mathbf{K n}=\mathbf{0 . 1 5}$ & $\left\langle v_{y}\right\rangle \mathrm{m} / \mathrm{s}$ & $\sigma_{v_{y}} \mathrm{~m} / \mathrm{s}$ \\
\hline DSMC & 10.1 & 238.6 \\
\hline LB2DSMC & 10.5 & 239.3 \\
\hline $\mathbf{K n}=\mathbf{0 . 2 5}$ & $\left\langle v_{y}\right\rangle \mathrm{m} / \mathrm{s}$ & $\sigma_{v_{y}} \mathrm{~m} / \mathrm{s}$ \\
\hline DSMC & 12.8 & 238.8 \\
\hline LB2DSMC & 13.4 & 239.6 \\
\hline \hline
\end{tabular}

Table 4: Comparison of the means and standard deviations of the distributions of Figures 8 expressed in DSMC units.

condition has been reached. The deviations between the means, about $4 \%$ for the case at $\mathrm{Kn}=0.15$ and about $5 \%$ for the case at $\mathrm{Kn}=0.25$, are in line with 

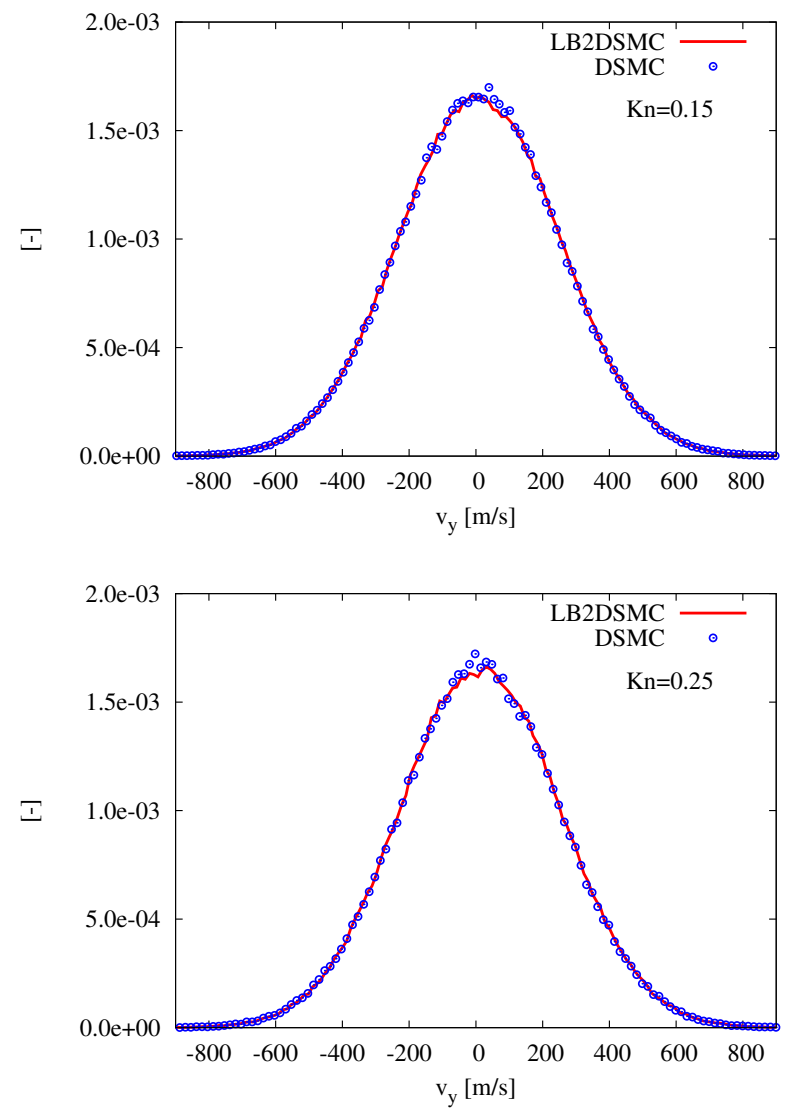

Figure 8: Distribution functions for the y-component, $v_{y}$, of the velocity of the particles, expressed in DSMC units, as obtained from the native DSMC simulation and from the reconstruction mapping scheme using the algorithm LB2DSMC outlined in Table 2 for $\mathrm{Kn}=0.15$ (top) and $\mathrm{Kn}=0.25$ (bottom), for the cell identified in Figure 5 
the deviations that are present in Figure 3 The standard deviations of the two distributions differ for about $0.3 \%$ for both the cases. Related to this, it has to be recalled that the temperature of Eq. (21) is the reference temperature imposed in the DSMC simulation. The magnitudes of these standard deviations are compatible with the reference temperature $(\mathrm{T}=273 \mathrm{~K})$ and the molecular mass $\left(m=6.63 \cdot 10^{-26} \mathrm{~kg}\right.$ ) for the gas used in the DSMC simulation.

It is important, however, that also the distributions of the realizations of the fluid velocity as obtained from DSMC and from the reconstruction mapping scheme match with each other. This is checked computing the fluid velocities as the instantaneous average velocity from all the velocities of particles residing in the chosen cell at regular time intervals (samples are taken once every 50 time steps) so to have uncorrelated samples. Also in this case, data are collected once the flow has reached a steady-state condition.

In Figure 9, the fluid velocity distributions are plotted for the case $\mathrm{Kn}=0.15$. Both the mean and standard deviations of the distributions obtained from the two methods are in good agreement, demonstrating that the LB2DSMC reconstruction step correctly maps the discrete LB distribution functions into the velocities of the DSMC particles.

\section{Hybrid model application}

As a proof of concept of a prospective LB-DSMC coupling, we applied a hybrid model to a plane Poiseuille flow with $\mathrm{Kn}=0.05$ and $\mathrm{Ma}=0.1$, based on centerline velocity.

In Figure 10, the geometry for the application of the hybrid method is drawn. The domain is divided into two subdomains. In each subdomain, one solution method is applied. In particular, we assume that, at a section located at $y=L_{0} / 2$, the two subdomains overlap and this buffer layer is composed by one cell along the flow direction and extends across the whole height $\mathrm{H}$ of the channel.

For simplicity, since we wanted to set up the functionality of the coupling, we 


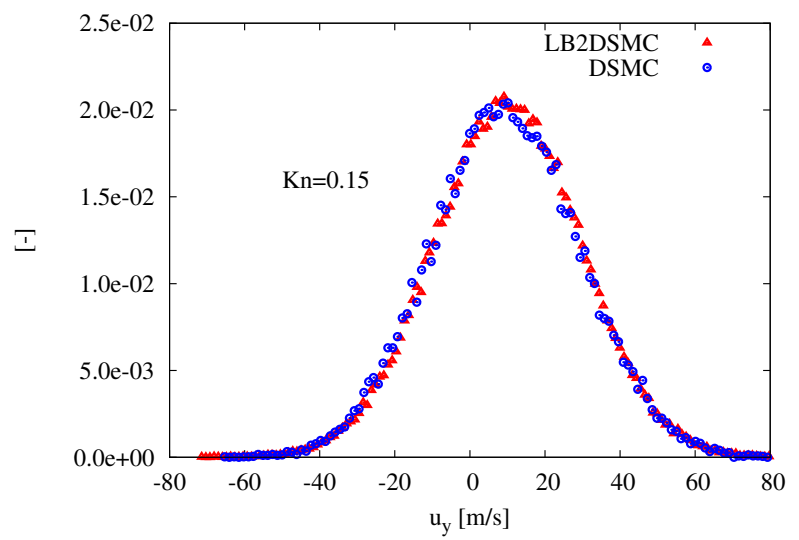

Figure 9: Fluid velocity distribution functions for the y-component, $u_{y}$, expressed in DSMC units, as obtained from the native DSMC simulation and from the reconstruction mapping scheme using the algorithm LB2DSMC outlined in Table 2 for $\mathrm{Kn}=0.15$, for the cell identified in Figure 5

use a D3Q19 LB model with kinetic boundary conditions and no regularization. The mapping scheme, also, is simpler than the one proposed in Section 2 In particular, we imposed that, at the centers of DSMC cells/LBM lattice sites within the buffer layer, the local equilibria are evaluated according to the hydrodynamic moments computed from the DSMC solution.

Operatively, we set the discrete equilibrium distribution functions, $f_{\mathrm{DSMC} 2 \mathrm{LB}, a}^{(0)}$, within the buffer layer as:

$$
\begin{aligned}
f_{\mathrm{DSMC} 2 \mathrm{LB}, a}^{(0)}= & w_{a} \rho_{\mathrm{DSMC}}\left[1+\frac{\boldsymbol{\xi}_{a} \cdot \mathbf{u}_{\mathrm{DSMC}}}{c_{s}^{2}}\right. \\
& \left.+\frac{\left(\boldsymbol{\xi}_{a} \cdot \mathbf{u}_{\mathrm{DSMC}}\right)^{2}}{2 c_{s}^{4}}-\frac{u_{\mathrm{DSMC}}^{2}}{2 c_{s}^{2}}\right]
\end{aligned}
$$

In Figure 11, we plot the evolution in time of the velocity profiles obtained from the hybrid method for the test previously introduced.

The three plotted profiles represent the data at the three sections along the channel located at $y=L_{0} / 4, y=L_{0} / 2$ and $y=3 L_{0} / 4$. The section at $y=L_{0} / 4$ is within the DSMC subdomain, while the section at $y=L_{0} / 2$ coincides with the

buffer layer position and the section at $y=3 L_{0} / 4$ is within the LBM subdomain. 


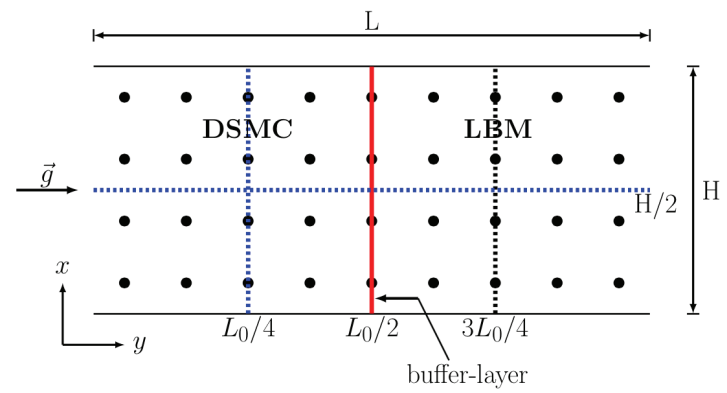

Figure 10: Schematic picture of the hybrid model application domain. The coupling occurs at the LBM lattice sites/DSMC cells placed at $y=L_{0} / 2$. The buffer layer comprises only one cell in the streamwise direction and all the cells along the transversal direction. The positions $y=L_{0} / 4$ and $y=3 L_{0} / 4$ identify the streamwise positions where the velocity profiles plotted in Figure 11 are evaluated.

From the plots of Figure 11, it is possible to see that the inherent statistical noise of the DSMC solution is transferred to the LBM velocity profiles. While averaging over time, this noise is reduced and also the LBM solution becomes accordingly, smoothened. Note that, in the DSMC solver, no particular means to reduce statistical noise, such as variance-reduction methods, 555 [57, has been adopted. Thus, there is certainly room for significant future improvements.

From inspection of Figure 11, it is possible to detect deviations between the DSMC velocity profile $\left(y=L_{0} / 4\right)$ and the LBM velocity profile $\left(y=3 L_{0} / 4\right)$ when the steady state is reached (see the plot at $t=1600$ ). These deviations can be attributed to the limitations of both the LB model and mapping scheme adopted in this test, as all the non-equilibrium effects have been discarded. The deviations will be removed by adopting the LB model able to extend the range of applicability of the LBM to rarefied gas flows and by including nonequilibrium effects in the passage of information betweeen the DSMC and the LBM as described in Section 2 2 This fully non-equilibrium hybrid model is under development. 

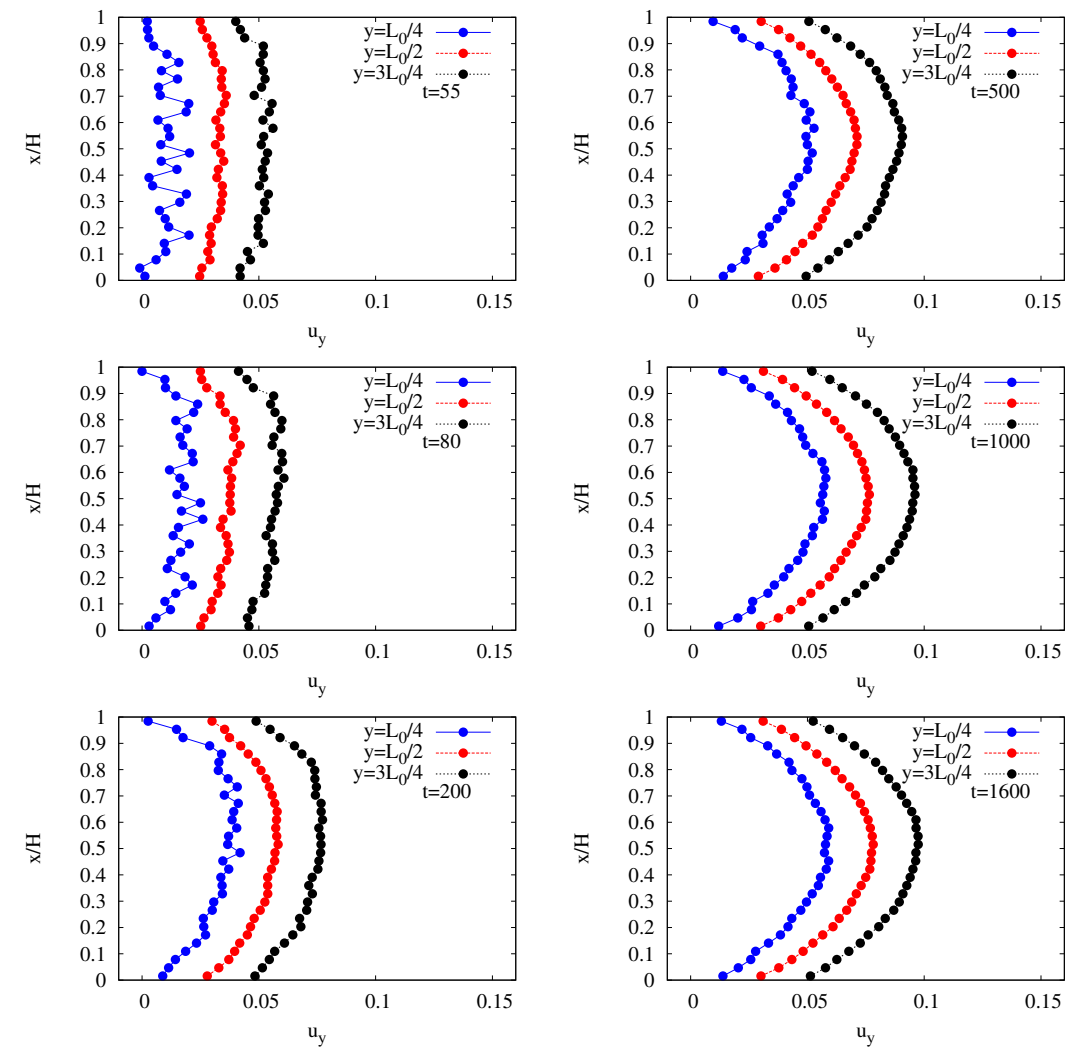

Figure 11: Evolution in time of the flow when the simplified mapping scheme DSMC2LB is activated at $y=L_{0} / 2$. Velocity profiles are plotted in correspondence of the channel sections identified in Figure 10 The velocity profiles, expressed in lattice units, at $y=L_{0} / 2$ and $y=3 L_{0} / 4$ are shifted +0.02 and +0.04 , respectively, to allow better visualization. 


\subsection{Breakdown parameter for the hybrid model}

The determination of the location where the coupling between the two methods is performed should be based on the identification of a breakdown parameter able to detect when the local non-equilibrium effects can no longer be accomodated within the given LB scheme. In the literature related to existing hybrid models, several definitions of the breakdown parameter are offered. The vast majority of these is based on the evaluation of the local density gradient, see e.g. 14. This choice is justified by the fact that those hybrid models are generally

330 applied to compressible flows problems. Such type of parameter, however, is not suitable for the LB method as, in its usual formulation, which is also adopted in this work, LB is a solver for weakly compressible flows. It is therefore needed to determine a different way to measure departure from the Navier-Stokes level of description (or Burnett, or even Super-Burnett level of description, if higherorder lattices are used).

While on a practical ground, it appears appropriate to place the interface between the two methods at least one mean free path away from the walls, a more rigorous approach might be based on the evaluation and comparison, between the LB and DSMC, of the coefficients of the Grad's expansion related to higher order moments, namely from the $2 n d$ order on, since these are no longer associated with microscopic invariants.

The implementation of such switching criteria, that should also take into due account the presence of fluctuations in the DSMC hydrodynamic moments, and which requires additional verification, will be object of future work.

\section{Computational efficiency}

We conclude by comparing the computational efficiency of the two methods and by estimating the computation times of the hybrid method with respect to a full DSMC simulation.

Both the DSMC and LBM codes are parallelized. All data presented in this section are from simulations run on a dual-core PC (Intel Core i5-6300U 2.4 
$\mathrm{GHz}$ ) with hyperthreading enabled and refer to the test cases of Sections 3.1 and 4 .

In Figure 12, the wall-clock time per computational time step is plotted as a function of the flow Kn number for both LBM models (D3Q19 and D3Q39 with the regularization procedure and kinetic boundary conditions) and DSMC. The wall-clock time per time step is constant for the LBM simulations while it shows a dependence on Kn for the DSMC method.

This might be explained considering that in DSMC, as kinetic theory prescribes, the total number of interparticle collisions scales with the number density. For the NoTimeCounter (NTC) algorithm, [ 6 , as the one employed here, one has:

$$
N_{\text {collis }}\left(t_{k}, x_{j}\right)=\frac{1}{2} N \bar{N} F_{N}\left(\sigma_{T} c_{r}\right)_{\max } \Delta t / V_{c}
$$

where $N=n\left(t_{k}, x_{j}\right) V_{c} / F_{N}$, with $n$ number density at time $t_{k}$ and cell $j, V_{c}$ the cell volume and $F_{N}$ the number of real molecules represented by a simulated computational particle, $\bar{N}=\langle N\rangle, \sigma_{T}$ the molecular cross section, $c_{r}$ the relative velocity between the selected particles to undergo collision, $\Delta t$ is the time step duration. In Eq. (28), the term $\left(\sigma_{T} c_{r}\right)_{\max }$ is the maximum value of the product between the collision cross section and the relative velocity between the selected particles in each grid cell.

From Eq. (28) the larger the Kn number, the smaller the number density $n$ and the smaller the total number of collisions. However, since the same number of cells and particles are used for all the simulations and since the collision step in the DSMC method is just one part of the algorithm, only a small decrease in the wall-clock time of the single computational time step is achieved while increasing Kn number. In the LBM, instead, Kn number determines the relaxation time $\tau$ but different values of $\tau$ do not affect the computational efficiency of the single computational step.

From Figure 12, it is also evident the fact that LBM wall-clock times are smaller than the ones for DSMC. In particular, a single computational time step for the D3Q19 model is 5 times faster and for the D3Q39 model is 2 times faster than for the DSMC. 
These numbers, however, do not tell the full story because DSMC is intrinsically characterized by statistical noise due to thermal fluctuations. This greatly affects the computational efficiency of the DSMC in comparison with LBM. In fact, to reduce the statistical noise on DSMC hydrodynamic moments, time (or ensemble) averaging is needed. For example, one standard deviation on the fluid velocity components measurement, $\sigma_{u_{i}}$, is given (at equilibrium) by [49]:

$$
\sigma_{u_{i}}=\sqrt{\frac{k_{B}\langle T\rangle}{m\langle N\rangle}} \frac{1}{\sqrt{S}}
$$

where $\langle T\rangle$ and $\langle N\rangle$ are the averages of temperature and number of computational particles in a cell and $\mathrm{S}$ is the number of independent statistical samples. An estimate on the statistical error on the evaluation of the fluid velocity is given as:

$$
\begin{array}{r}
E_{u_{i}}=\frac{\sigma_{u_{i}}}{\left|\left\langle u_{i}\right\rangle\right|}=\sqrt{\frac{k_{B}\langle T\rangle}{m\langle N\rangle}} \frac{1}{\sqrt{S}} \frac{1}{\left|\left\langle u_{i}\right\rangle\right|} \\
=\frac{1}{\sqrt{\gamma\langle N\rangle S \mathrm{Ma}}}
\end{array}
$$

where $\gamma$ is the gas specific heat ratio (1.67 for Argon) and Ma is the Mach number.

If a $1 \%$ fractional error is desired, for a $\mathrm{Ma}=0.1$ flow and $\langle N\rangle=100, S \approx 3600$ independent samples are needed. Generally, to obtain independent samples 10100 time steps between the samples are required. In all the simulations in this work, we decided to perform the sampling every 50 time steps. Calculation of the correlation coefficients between sampled quantities showed that, for the flow of these tests, a 50 time steps interval is sufficient, e.g.:

$$
\operatorname{corr}\left(u_{x}, u_{y}\right)=\frac{\left\langle\delta u_{x} \delta u_{y}\right\rangle}{\sqrt{\left\langle\delta u_{x}^{2}\right\rangle\left\langle\delta u_{y}^{2}\right\rangle}}=-0.008 .
$$

Estimates on the number of needed independent samples to reach a given fractional error and on the size of the time steps interval so to obtain independent samples allow to determine the number of the required total computational time steps. So, for the tests we performed, at least 180000 time steps are needed. 

steps. About 5000 time steps are sufficient to reach the final solution.

These numbers directly reflect in the comparison between the total wall-clock times needed for the DSMC and LBM simulations. Using data collected in Figure 12, if the DSMC is compared with the D3Q19 model, then the latter is about the latter is about 70 times faster. Moreover, a reduction in the total number of DSMC particles guaranteed by reducing the domain assigned to the DSMC reflects in a reduction in the wall-clock time per time step as shown in Figure 13 where a linear scaling is found for the range of particles typically employed for the flow under consideration.

Finally, these numbers allow to estimate the potential gain in efficiency that can be obtained by the application of the hybrid model.

Using the simplified mapping scheme to pass from DSMC to LBM as described in Section 4 and assuming that the domain is divided into two subdomains of equal size, then a speed-up of about 1.7 with respect to a full DSMC simulation over the whole domain is reachable for the tested Poiseuille flow. To be noted that the over-head due to the application of the simplified mapping scheme is very limited since the buffer layer is composed of just one layer of cells/lattice nodes. For more complicated flows, however, coupling may be required to be applied over larger overlapping zones.

\section{Conclusions}

We developed a kinetic mapping scheme based on Grad's moments method and Gauss-Hermite quadrature in view of coupling DSMC and LBM models to simulate isothermal flows with non-uniform rarefaction effects. The main steps

of the mapping algorithm between DSMC and LBM in order to allow an accurate passage between the two methods domains were discussed. To extend the range of applicability of LBM beyond the Navier-Stokes equation level, and thus postponing the passage to the DSMC solver, the need for adopting a high-order 


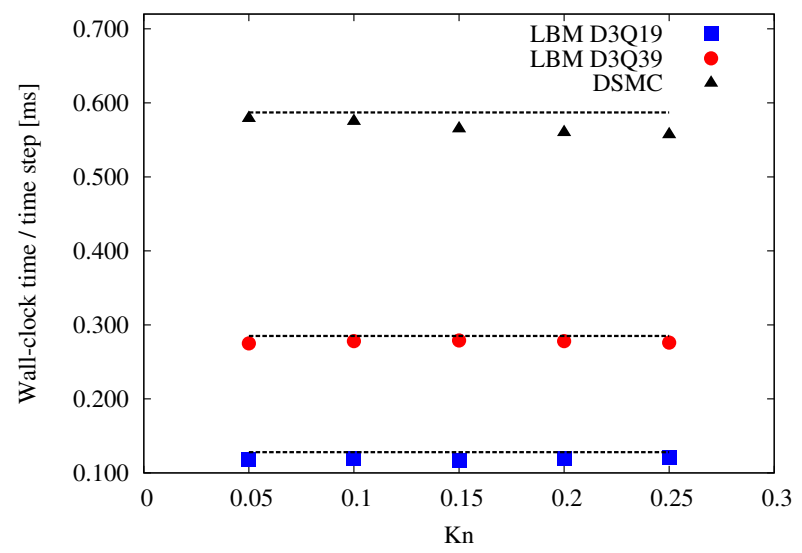

Figure 12: Wall-clock time per computational time step in LBM D3Q19, LBM D3Q39 and DSMC for the test of Section 3.1. While LBM data wall clock time does not depend on Kn number, DSMC data show a mild dependence on Kn. Note that both LBM and DSMC simulations, as stated in Section 3.1 are run on a grid based on the requirements for the DSMC simulation at $\mathrm{Kn}=0.05$ and kept the same for all the simulations at different $\mathrm{Kn}$ number. 32000 particles are employed for the DSMC simulations.

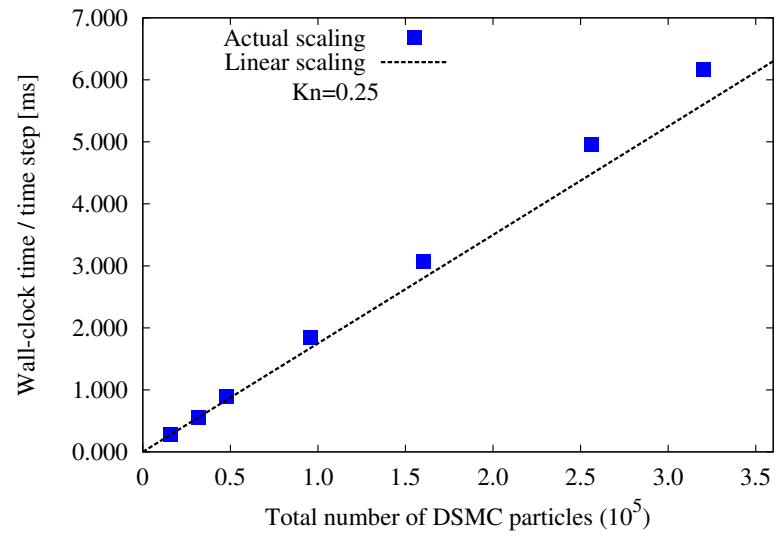

Figure 13: Wall-clock time per time step for DSMC as a function of the total number of particles for a simulation at $\mathrm{Kn}=0.25$. Linear scaling is shown for the range of particles per cell typically employed in DSMC simulations. Savings in the total number of particles reflect in a linear reduction of the total wall-clock time of the simulation. The simulated flow is the same presented in Section 3.1 
lattice (D3Q39) and a regularization procedure for the LBM is demonstrated by

Poiseuille flow up to $\mathrm{Kn}=0.25$. As a proof of concept of the hybrid method, a simpler version of the mapping scheme which enforces the passage through local equilibrium states has been performed for the simulation of a plane Poiseuille flow at $\mathrm{Kn}=0.05$. We have also estimated that the adoption of the hybrid scheme

\section{Acknowledgements}

This research is supported by the Dutch Technology Foundation STW, which is part of the Netherlands Organisation for Scientific Research (NWO), and which is partly funded by the Ministry of Economic Affairs. One of the authors (SS) was partially supported by the Integrated Mesoscale Architectures for Sustainable Catalysis (IMASC), an Energy Frontier Research Center funded by the US Department of Energy, Office of Science, Basic Energy Sciences under Award No. DE-SC0012573, during his stay at the Rowland Institute for Science at Harvard University.

\section{Appendix A. Scaling factors}

To be able to apply the proposed methods also in engineering contexts and in parallel with experiments, we decided to employ in the DSMC simulations dimensional variables with SI units. This implies that, prior to any transfer of information between LB and DSMC, a proper conversion from lattice units to SI units, or vice versa according to the fact that the DSMC2LB or LB2DSMC 405 mapping scheme is involved, has to be performed.

The basic elementary conversion scales are here introduced: 


\section{- Length scale}

Since in LB we assume the lattice spacing $\Delta x$ as the space unit and since we impose that the centers of the DSMC cells overlap with the LB sites, then the length scale is set as:

$$
L_{0}=\Delta x_{\mathrm{DSMC}} \quad[\mathrm{m}],
$$

where $\Delta x_{\mathrm{DSMC}}$ is the linear distance between the centers of two adjacent DSMC cells. Note that this implies that, at least in the buffer layer, the DSMC cells are cubic;

- Time scale

Similarly, the time unit within the LB simulation is the elementary lattice time-step. The physical value can be defined through the speed of sound within the lattice, $c_{s}$, and of the gas in the DSMC simulation, $a$, as

$$
T_{0}=\frac{c_{s}}{a} \Delta x_{\mathrm{DSMC}} \quad[\mathrm{s}] .
$$

- Mass scale

As the mass within the DSMC cells/LB nodes where coupling occurs must be conserved, and assuming the lattice particles are given a unit mass, then the mass scale can be defined as follows:

$$
M_{0}=\frac{F_{N, \mathrm{DSMC}} N_{\mathrm{DSMC}} m}{\sum_{a} f_{\mathrm{LB}, a}}[\mathrm{~kg}],
$$

\section{Appendix B. Hermite polynomials}

The $n$-th order Hermite polynomial is defined according to Rodrigues' formula, [58, as:

$$
\mathcal{H}^{(n)}(\boldsymbol{\xi})=\frac{(-1)^{n}}{\omega(\boldsymbol{\xi})} \nabla^{n} \omega(\boldsymbol{\xi})
$$


which is a rank- $n$ tensor and a polynomial of degree $n$ in $\boldsymbol{\xi}$. In Eq. B.1 $\omega(\boldsymbol{\xi})$ is the weight function associated with the Hermite polynomials in $D$-dimensional Cartesian coordinate $\boldsymbol{\xi}$ :

$$
\omega(\boldsymbol{\xi})=\frac{1}{(2 \pi)^{D / 2}} \exp \left(-\frac{\xi^{2}}{2}\right) .
$$

The first Hermite polynomials, therefore, read as:

$$
\begin{aligned}
& \mathcal{H}^{(0)}(\boldsymbol{\xi})=1, \\
& \mathcal{H}_{i}^{(1)}(\boldsymbol{\xi})=\xi_{i}, \\
& \mathcal{H}_{i j}^{(2)}(\boldsymbol{\xi})=\xi_{i} \xi_{j}-\delta_{i j}, \\
& \mathcal{H}_{i j k}^{(3)}(\boldsymbol{\xi})=\xi_{i} \xi_{j} \xi_{k}-\xi_{i} \delta_{j k}-\xi_{j} \delta_{i k}-\xi_{k} \delta_{i j} .
\end{aligned}
$$

\section{References}

[1] C.-M. Ho and Y.-C. Tai, "Micro-electro-mechanical-systems (mems) and fluid flows," Annu. Rev. Fluid Mech., vol. 30, pp. 579-612, 1998.

[2] J. M. Reese, M. A. Gallis, and D. A. Lockerby, "New directions in fluid dynamics: non-equilibrium aerodynamic and microsystem flows," Phil. Trans. R. Soc. Lond. A, vol. 361, pp. 2967-2988, 2003.

[3] G. Karniadakis, A. Beskok, and N. Aluru, Microflows and Nanoflows. Fundamentals and Simulation. New York: Springer-Verlag, 2005.

[4] I. D. Boyd, G. Chen, and G. V. Candler, "Predicting failure of the continuum fluid equations in transitional hypersonic flows," Phys. Fluids, vol. 7, p. $210,1995$.

[5] K. Huang, Statistical mechanics. New York: John Wiley \& Sons, 1987.

[6] G. A. Bird, Molecular gas dynamics and the direct simulation of gas flows. Oxford: Clarendon University Press, 1994.

430

[7] S. Chapman and T. G. Cowling, The mathematical theory of non-uniform gases: an account of the kinetic theory of viscosity, thermal conduction and diffusion in gases. Cambridge: Cambridge University Press, 3rd ed., 1970. 
[8] A. L. Garcia, J. Bell, W. Crutchfield, and B. Alder, "Adaptive mesh and algorithm refinement using Direct Simulation Monte Carlo," J. Comput. Phys., vol. 154, pp. 134-155, 1999.

[9] M. M. Mansour, F. Baras, and A. Garcia, "On the validity of hydrodynamics in plane Poiseuille flows," Physica A, vol. 240, pp. 255-267, 1997.

[10] W.-M. Zhang, G. Meng, and Z. Wei, "A review on slip models for gas microflows," Microfluid. Nanofluid., vol. 13, pp. 845-882, 2012.

[11] H. Grad, "On the kinetic theory of rarefied gases," Commun. Pure Appl. Math., vol. 2, pp. 331-407, 1949.

[12] H. Struchtrup, Macroscopic transport equations for rarefied gas flows: Approximation methods in kinetic theory. Heidelberg: Springer-Verlag, 2005.

[13] R. Roveda, D. B. Goldstein, and P. L. Varghese, "Hybrid Euler/particle approach for continuum/rarefied flows," J. Spacecraft Rockets, vol. 35, pp. 258-265, 1998.

[14] H. S. Wijesinghe, R. D. Hornung, A. L. Garcia, and N. G. Hadjiconstantinou, "Three-dimensional hybrid continuum-atomistic simulations for multiscale hydrodynamics," J. Fluids Eng., vol. 126, pp. 768-777, 2004.

[15] J.-S. Wu, Y.-Y. Lian, G. Cheng, R. P. Koomullil, and K.-C. Tseng, "Development and verification of a coupled DSMC-NS scheme using unstructured mesh," J. Comput. Phys., vol. 219, pp. 579-607, 2006.

[16] T. E. Schwatzentruber, L. C. Scalabrin, and I. D. Boyd, "A modular particle continuum numerical method for hypersonic non-equilibrium gas flows," J. Comput. Phys., vol. 225, pp. 1159-1174, 2007.

[17] D. A. Kessler, E. S. Oran, and C. R. Kaplan, "Towards the development of a multiscale, multiphysics method for the simulation of rarefied gas flows," J. Fluid Mech., vol. 661, pp. 262-293, 2010. 
[18] S. Pantazis and H. Rusche, "A hybrid continuum-particle solver for unsteady rarefied gas flows," Vacuum, vol. 109, pp. 275-283, 2014.

[19] K. Farber, P. Farber, J. Grabel, S. Krick, J. Reitz, and P. Ueberholz, "Development and validation of a coupled Navier-Stokes/DSMC simulation for rarefied gas flow in the production process for OLEDs." In press, 2015.

[20] J.-F. Bourgat, P. L. Tallec, and M. Tidriri, "Coupling Boltzmann and Navier-Stokes equations by friction," J. Comput. Phys., vol. 127, pp. 227$245,1996$.

[21] P. L. Tallec and F. Mallinger, "Coupling Boltzmann and Navier-Stokes equations by half-fluxes," J. Comput. Phys., vol. 136, pp. 51-67, 1997.

[22] A. L. Garcia and B. J. Alder, "Generation of the Chapman-Enskog distribution," J. Comput. Phys., vol. 140, pp. 66-70, 1998.

[23] H. A. Al-Mohssen, N. G. Hadjiconstantinou, and I. G. Kevrekidis, "Acceleration methods for coarse-grained numerical solution of the Boltzmann equation," J. Fluids Eng., vol. 129, pp. 908-912, 2007.

[24] P. Degond, J.-G. Liu, and L. Mieussens, "Macroscopic fluid models with localized kinetic upscaling effects," Multiscale Model. Simul., vol. 53, pp. 940979, 2006.

[25] S. Succi, "Mesoscopic modeling of slip motion at fluid-solid interfaces with heterogeneous catalysis," Phys. Rev. Lett., vol. 89, p. 064502, 2002.

[26] F. Toschi and S. Succi, "Lattice Boltzmann method at finite Knudsen numbers," Europhys. Lett., vol. 69, pp. 549-555, 2005.

[27] M. Sbragaglia and S. Succi, "Analytical calculation of slip flow in lattice Boltzmann models with kinetic boundary conditions," Phys. Fluids, vol. 17, p. 093602, 2005. 
[28] M. Sbragaglia and S. Succi, "A note on the lattice Boltzmann method beyond the Chapman-Enskog limits," Europhys. Lett., vol. 73, pp. 370$376,2006$.

[29] R. Zhang, Z. Shan, and H. Chen, "Efficient kinetic method for fluid simulation beyond the Navier-Stokes equation," Phys. Rev. E, vol. 74, p. 046703, 2006.

[30] S. Ansumali, I. V. Karlin, S. Arcidiacono, A. Abbas, and N. I. Pasianakis, "Hydrodynamics beyond Navier-Stokes: exact solution to the lattice Boltzmann method," Phys. Rev. Lett., vol. 95, p. 260605, 2007.

[31] X.-D. Niu, S.-A. Hyodo, T. Munekata, and K. Suga, "Kinetic lattice Boltzmann method for microscale gas flows: Issues on boundary condition, relaxation time, and regularization," Phys. Rev. E, vol. 76, p. 036711, 2007.

[32] G.-H. Tang, Y.-H. Zhang, and D. Emerson, "Lattice Boltzmann models for nonequilibrium gas flows," Phys. Rev. E, vol. 77, p. 046701, 2008.

[33] J. Meng and Y. Zhang, "Accuracy analysis of high-order lattice Boltzmann models for rarefied gas flows," J. Comput. Phys., vol. 230, pp. 835-849, 2011.

[34] J. Meng and Y. Zhang, "Gauss-Hermite quadratures and accuracy of lattice Boltzmann models for nonequilibrium gas flows," Phys. Rev. E, vol. 83, p. 036704, 2011.

[35] T. Reis and P. Dellar, "Lattice Boltzmann simulations of pressure-driven flows in microchannels using Navier-Maxwell slip boundary conditions," Phys. Fluids, vol. 24, p. 112001, 2012.

[36] X. Liu and Z. Guo, "A lattice Boltzmann study of gas flows in a long micro-channel," Comput. Math. Appl., vol. 65, pp. 186-193, 2013.

[37] S. Tao and Z. Guo, "Boundary condition for lattice Boltzmann modeling of microscale gas flows with curved walls in the slip regime," Phys. Rev. E, vol. 91, p. 043305, 2015. 
[38] S. Succi, The Lattice Boltzmann Equation for fluid dynamics and beyond. Oxford: Clarendon University Press, 2001.

[39] J. Zhang, "Lattice Boltzmann method for microfluidics: models and applications," Microfluid. Nanofluid., vol. 10, pp. 1-28, 2011.

[40] F. Verhaeghe, L.-S. Luo, and B. Blanpain, "Lattice Boltzmann modeling of microchannel flow in slip flow regime," J. Comput. Phys., vol. 228, pp. 147$157,2009$.

[41] Z. Guo, J. Qin, and C. Zheng, "Generalized second-order slip boundary condition for nonequilibrium gas flows," Phys. Rev. E, vol. 89, p. 013201, 2014.

[42] S. Ansumali and I. V. Karlin, "Kinetic boundary conditions in the lattice Boltzmann method," Phys. Rev. E, vol. 66, p. 026311, 2002.

[43] Z. Chai, Z. Guo, L. Zheng, and B. Shi, "Lattice Boltzmann simulation of surface roughness effect on gaseous flow in a microchannel," J. Appl. Phys., vol. 104, p. 014902, 2008.

[44] X. Shan, X.-F. Yuan, and H. Chen, "Kinetic theory representation of hydrodynamics: a way beyond the Navier-Stokes equation," J. Fluid Mech., vol. 550, pp. 413-331, 2006.

[45] A. Montessori, M. L. Rocca, G. Falcucci, and S. Succi, "Regularized lattice BGK versus highly accurate spectral methods for cavity flow simulations," Int. J. Mod. Phys. C, vol. 25, p. 1441003, 2014.

[46] Z. Guo, C. Zheng, B. Shi, and T. S. Zhao, "Thermal lattice Boltzmann equation for low Mach number flows: Decoupling model," Phys. Rev. E, vol. 75 , p. 036704, 2007.

[47] J. Meng, Y. Zhang, N. G. Hadjiconstantinou, G. A. Radtke, and X. Shan, "Lattice ellipsoidal statistical BGK model for thermal non-equilibrium flows," J. Fluid Mech., vol. 718, pp. 347-370, 2013. 
[48] P. Lallemand and L.-S. Luo, "Theory of the lattice Boltzmann method: Acoustic and thermal properties in two and three dimensions," Phys. Rev. E, vol. 68, p. 036706, 2003.

[49] N. G. Hadjiconstantinou, A. L. Garcia, M. Z. Bazant, and G. He, "Statistical error in particle simulations of hydrodynamic phenomena," J. Comput. Phys., vol. 187, pp. 274-297, 2003.

[50] G. H. Tang, W. Q. Tao, and Y. L. He, "Lattice Boltzmann method for gaseous microflows using kinetic theory boundary conditions," Phys. Fluids, vol. 17, p. 058101, 2005.

[51] G. H. Tang, Y. H. Zhang, X. J. Gu, and D. R. Emerson, "Lattice Boltzmann modelling Knudsen layer effect in non-equilibrium flows," Europhys. Lett., vol. 83 , p. 40008, 2008.

[52] M. A. Gallis, J. R. Torczynski, D. J. Rader, M. Tij, and A. Santos, "Normal solutions of the Boltzmann equation for highly nonequilibrium Fourier flow and Couette flow," Phys. Fluids, vol. 18, p. 017104, 2006.

[53] C. R. Lilley and J. E. Sader, "Velocity profile in the Knudsen layer according to the Boltzmann equation," Proc. R. Soc. A, vol. 464, pp. 2015-2035, 2008.

[54] X. Shan, "General solution of lattices for cartesian lattice Bhatnagar-GrossKrook models," Phys. Rev. E, vol. 81, p. 036702, 2010.

[55] C. R. Kaplan and E. S. Oran, "Nonlinear filtering for low-velocity gaseous microflows," AIP Conference Proceedings, vol. 585, pp. 472-479, 2001.

[56] J. Fan and C. Shen, "Statistical simulation of low-speed rarefied gas flows," J. Comput. Phys., vol. 167, pp. 393-412, 2001.

[57] L. L. Baker and N. G. Hadjiconstantinou, "Variance reduction for Monte Carlo solutions of the Boltzmann equation," Phys. Fluids, vol. 17, p. 051703, 2005. 
[58] P. J. Davis and P. Rabinowitz, Methods of numerical integration. New York: Academic Press, 1975. 